\title{
Galectin-3 and PTEN Expression in Pancreatic Ductal Adenocarcinoma, Pancreatic Neuroendocrine Neoplasms and Gastrointestinal Tumors on Fine-Needle Aspiration Cytology
}

\author{
Kun Jiang Diane Lawson Cynthia Cohen Momin T. Siddiqui \\ Department of Pathology and Laboratory Medicine, Emory University School of Medicine, Atlanta, Ga., USA
}

\author{
Key Words \\ Galectin-3 · Phosphatase and tensin homolog · Fine-needle \\ aspiration - Adenocarcinoma · Neuroendocrine tumor . \\ Gastrointestinal tumor
}

\begin{abstract}
Objective: Galectin-3 has been implicated in the carcinogenesis of pancreatic ductal adenocarcinoma (PDAC). Its applicability in pancreatic fine-needle aspiration (FNA) in separating malignant from benign lesions has never been addressed. In addition, a correlation between Galectin-3 and tumor suppressor phosphatase and tensin homolog (PTEN) and their potential diagnostic value has never been tested. Study Design: This study analyzed Galectin-3 immunohistochemical expression in FNA cell blocks of PDAC, pancreatic neuroendocrine neoplasms (PNEN), gastrointestinal stromal tumors (GIST) and non-tumor pancreatic tissue. In parallel, Galectin-3 and PTEN levels were evaluated in a tumor tissue microarray (TMA). $\boldsymbol{R} \boldsymbol{e}$ sults: Forty-four of 46 PDACFNA and 32 of 33 PDAC TMA demonstrated tumor-specific Galectin-3 positivity. In contrast, Galectin-3 was not detected in PNEN and GIST. Total loss of PTEN was displayed by 26 of 33 PDAC, while non-neoplastic tissues all retained PTEN expression. Conclusion: Galectin- 3 could be a valuable marker to help diagnose PDAC and rule out PNEN and GIST. In addition, PTEN positivity strongly argues against a diagnosis of PDAC. These data also advocate their potential diagnostic roles in the work up of challenging cytologic cases requiring ancillary test confirmation.

(c) 2014 S. Karger AG, Basel
\end{abstract}

\section{KARGER}

E-Mail karger@karger.com

www.karger.com/acy

\section{Introduction}

Galectin-3 is a member of the lectin-like galectin family of glycoprotein-binding proteins. All the members of this family are characterized by conserved peptide sequences in their carbohydrate recognition domains (CRDs), which bind $\beta$-galactoside-containing glycoconjugates $[1,2]$. Most galectins are multivalent, being intrinsically composed of two CRDs or existing in a dimerized form; uniquely, Galectin-3 is composed of an N-terminal, non-lectin domain consisting of multiple repeats of a peptide sequence rich in proline, glycine and tyrosine, in addition to its C-terminal CRD [3-5]. Both the C-terminal CRD domain and the $\mathrm{N}$-terminal non-lectin domain of Galectin-3 are essential for its role in signal transduction, cellular adhesion and lattice formation $[2,6-8]$.

Galectin- 3 has been implicated in various biological and pathophysiological processes, such as cell proliferation, transformation, migration, tumor cell invasion and cell-matrix adhesion $[1,2,4,9,10]$. It exists in several cellular compartments, including the cytoplasm, the cell surface, the nucleus and secreted form [2]. The impact of Galectin-3 has also been evaluated in different cancers $[6-8,11,12]$. It has been reported that Galectin-3 mRNA is upregulated in pancreatic cancer [13-15], and increased in chronic pancreatitis, and may be involved in extracellular matrix changes [16].

Pancreatic ductal adenocarcinoma (PDAC) is one of the major leading causes of cancer-related death, mainly affecting patients aged between 50 and 70 years. Due to its in- 
sidious onset, aggressive behavior and the absence of a sensitive detection method, its early metastatic properties and lack of effective therapies, the five-year survival rate for PDAC remains less than 5\% [17]. In terms of the molecular events underlying the pathogenesis of PDAC, mutations in $\mathrm{K}$-Ras are found in the majority of cases [18]. Although key roles of K-Ras mutations have been proposed to contribute in the development of PDAC [19], they cannot fully account for, or be solely resorted to, in diagnosing early PDAC, as K-Ras mutations are also found in chronic pancreatitis and even normal tissues [20]. Moreover, the mechanisms by which K-Ras is further activated still remain incompletely understood. Diagnosis of PDAC can also be challenging in small samples obtained from fine-needle aspiration (FNA) samples. In addition, immunohistochemical analysis may also be required to differentiate PDAC from pancreatic neuroendocrine neoplasms (PNEN) and gastrointestinal stromal tumor (GIST) samples.

In this study, we evaluated the expression of Galectin-3 in FNA specimens of pancreatic tissue, including benign pancreas, pancreatitis, PDAC, PNEN and GIST by immunohistochemistry. In addition, we also studied Galectin-3 and phosphatase and tensin homolog (PTEN) expression in PDAC tissue microarrays (TMAs) prepared from pancreatic Whipple resection specimens, so as to confirm the data obtained from pancreatic FNA analyses. Our goal was to determine whether there are specific alterations of Galectin-3 and PTEN in pancreatic neoplasms, especially PDAC, and whether we could utilize immunohistochemical stains as potential tumor markers in our future endeavor of diagnosing early PDAC in FNA samples.

\section{Materials and Methods}

\section{FNA Study Design}

This retrospective cohort study was approved by the Institutional Review Board of Emory University. All patients provided written informed consent for endoscopic ultrasound (EUS) with or without FNA. EUS procedures utilizing FNA were selected. Cases involving FNA of pancreatic cysts were excluded. Patients with predominantly solid lesions on cross-sectional imaging or with mass lesions (with or without a cystic component) were included. FNA data were collected for patients undergoing EUS-FNA between January 2011 and October 2012. Patient demographics, date and indication of EUS procedure, endoscopist, anatomic site and final cytopathologic results were obtained from the electronic medical records and endoscopy documentation software, and were compiled into a database.

\section{EUS-FNA and ROSE}

The EUS procedures were performed with the patient under monitored anesthesia care or conscious sedation. All EUS-FNA specimens were obtained by 1 of 2 experienced echoendosonogra- phers using curvilinear echoendoscopes (Olympus, Center Valley, Pa., USA). FNA samples were obtained during EUS-FNA using 25-, 22- and 19-gauge EUS-FNA needles. In our institution, ROSE (rapid on-site evaluation) is commonly utilized for mass lesions of the pancreas. Corresponding EUS-FNA slides are immediately Diff-Quik (Thermo-Fisher Scientific, Kalamazoo, Mich., USA) stained and evaluated for adequacy. The remainder of the FNA specimen was submitted for the preparation of cell blocks (CB) by fixing in $10 \%$ neutral-buffered formalin and then poured undiluted into a 15 - $\mathrm{ml}$ glass centrifuge tube coated with collodion solution and centrifuged for $7 \mathrm{~min}$. The supernatant was discarded and the resultant collodion bag (JT Baker, Phillipsburg, N.J., USA) and centrifuged material underwent routine paraffin embedding as a $\mathrm{CB}$ followed by staining with hematoxylin and eosin (HE).

\section{Tissue Microarrays}

Tissue Microarrays (TMAs) were prepared from pancreatic Whipple specimens with a diagnosis of PDAC. Donor blocks from PDAC cases were selected. A morphologically representative area of interest within the donor block was identified under the microscope using an HE-stained section on a glass slide. Two 1-mm cores from each block were removed from the donor and inserted into a recipient paraffin block using a precise spacing pattern.

\section{Case Selection}

Forty-six PDAC, 8 non-neoplastic pancreases, including 3 chronic pancreatitides, 5 PNEN and 13 GIST CB were selected for this study. Additionally, TMAs of 36 PDAC cases were included.

\section{Antibodies, Reagents and Immunohistochemistry}

Anti-Galectin-3 rabbit polyclonal antibody [1:100 (clone 9C4); RDI, Flanders, N.J., USA] and anti-PTEN rabbit monoclonal antibody (1:100; Cell Signaling Technology Inc., Beverly, Mass., USA) were used to evaluate Galectin-3 and PTEN expression immunohistochemically. The immunohistochemical procedures were carried out based on the manufacturers' recommendations, using the Dako Autostainer (Dako, Carpenteria, Calif., USA).

Immunohistochemical staining was performed on $5-\mu \mathrm{m}$ sections after antigen retrieval in citrate buffer $\mathrm{pH} 6.0$ and endogenous peroxidase blocking. The sections were then incubated with antibody against Galectin-3 or PTEN for $30 \mathrm{~min}$ at room temperature, and then incubated with secondary antibody at room temperature for $30 \mathrm{~min}$. EnVision Dual Link kit (Dako), which uses a polymer, was the detection method, with diaminobenzidine as the chromogen, and hematoxylin as the counterstain.

Known positive controls (human thyroid papillary carcinoma for Galectin-3, endometrial adenocarcinoma for PTEN) and negative controls with antibodies replaced with buffer were run in parallel. Membranous and granular cytoplasmic expression of Galectin-3 ( $3+$ in at least $10 \%$ of cells) was considered positive. A strongto-moderate nuclear signal with at least moderate cytoplasmic staining in at least $50 \%$ of cells was considered positive for PTEN. The staining results were evaluated by two pathologists (K.J. and M.S.) based on the intensity and percentage of staining in tumor cells, with agreement reached.

\section{Statistical Analysis}

The significance of differences between the groups was judged using a two-tailed Student $t$ test. Results were considered statistically significant if the $\mathrm{p}$ value was $<0.05$. 
Fig. 1. Challenging FNA cases of pancreatic neoplasms. Two cases of poorly differentiated PDAC (a, b), PNEN (c) and GIST (d).
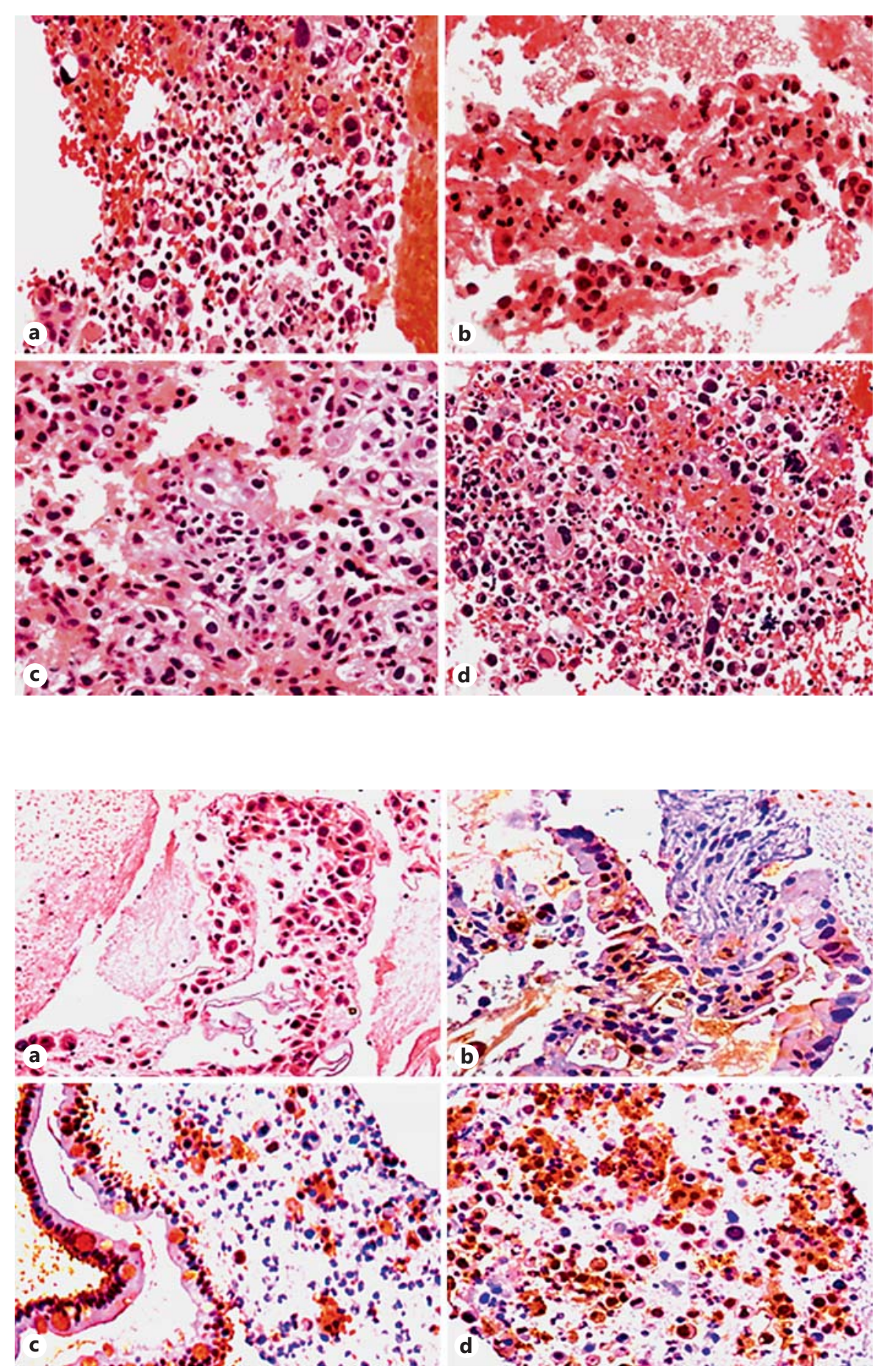

Fig. 2. Immunohistochemical study of $\mathrm{Ga}$ lectin-3 levels in FNA specimens of PDAC. a FNA CB slide of a poorly differentiated PDAC. b-d Strong, positive stains of Galectin-3 in well, moderately and poorly differentiated PDAC. Occasional inflammatory cells are also stained positive. Note the presence of positivity in mixed small intestine epithelium (c), which serves as an internal control.

\section{Results}

All pancreatic FNA smears and CB (fig. 1a-d) were reviewed. In PDAC specimens, out of 46 FNA CB, 44 (95.7\%) demonstrated significant tumor cell-specific Galectin-3 positivity (fig. $2 \mathrm{a}-\mathrm{d}$ ). The remaining 2 (4.3\%) CB were negative. Galectin-3 expression was significantly higher in PDAC than the non-neoplastic pancreatic tissue samples, as only $1 \mathrm{CB}(12.5 \%)$ from the 8 non-neoplastic cases showed focal, mild-to-moderate Galectin-3 staining $(2+; \mathrm{p}<0.001$; fig. 2 ; sensitivity $95.7 \%$; specificity $87.5 \%$; $<<0.001)$. The remaining 7 non-neoplastic CB 
Table 1. Expression of Galectin-3 in FNA CB of PDAC and benign tissue

\begin{tabular}{lll}
\hline Tissue type & $\begin{array}{l}\text { Galectin-3 } \\
\text { positive }\end{array}$ & $\begin{array}{l}\text { Galectin-3 } \\
\text { negative }\end{array}$ \\
\hline PDAC $(\mathrm{n}=46)$ & $44(95.7)$ & $2(4.3)$ \\
Benign $(\mathrm{n}=8)$ & $1(12.5)$ & $7(87.5)$ \\
\hline
\end{tabular}

Values in parentheses are percentages. $\mathrm{p}<0.001$.

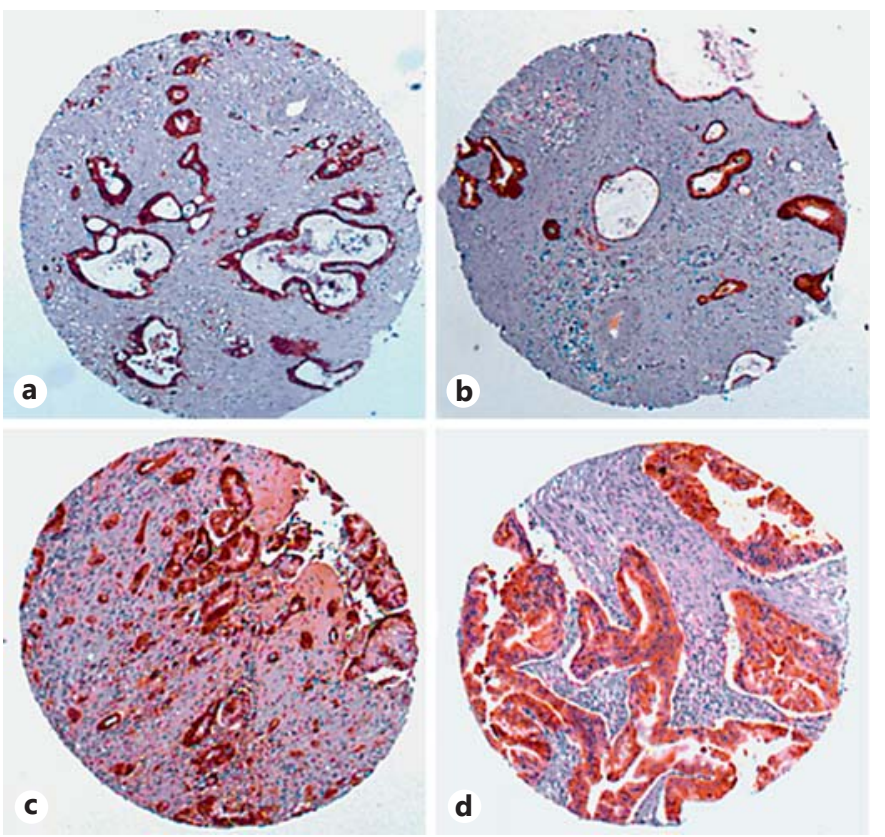

Fig. 3. Immunohistochemical study of Galectin-3 levels in a tissue microarray of PDAC. Unique and significant expression of Galectin-3 is demonstrated in PDAC (a-d), with minimal-to-negative levels in adjacent non-tumor tissue and normal pancreas (b). Positive staining of Galectin-3 is detected in well $(\mathbf{a}, \mathbf{b})$, moderately $(\mathbf{d})$ and poorly differentiated (c) PDAC. Occasional inflammatory cells stained positive (c).

did not show immunoreactivity for Galectin-3 staining (table 1).

In pancreatic TMAs, Galectin-3 was universally upregulated in PDAC (table 2); 32 of 33 (96.97\%) PDAC demonstrated strong $(3+)$ positive Galectin-3 staining. The remaining 1 PDAC was negative. The 3 non-neoplastic cases all showed negativity for Galectin-3 (sensitivity 96.97\%; specificity $100 \%$; $\mathrm{p}<0.001$; fig. $3 \mathrm{a}-\mathrm{d}$ ).

These findings demonstrated that Galectin-3 is uniquely upregulated in PDAC in both $\mathrm{CB}$ and TMA with
Table 2. Expression of Galectin-3 in TMA of PDAC and non-neoplastic pancreas

\begin{tabular}{lll}
\hline & $\begin{array}{l}\text { Galectin-3 } \\
\text { positive }\end{array}$ & $\begin{array}{l}\text { Galectin-3 } \\
\text { negative }\end{array}$ \\
\hline PDAC $(\mathrm{n}=33)$ & $\begin{array}{l}32(96.97) \\
0(0)\end{array}$ & $\begin{array}{l}1(3.03) \\
3(100)\end{array}$ \\
Non-neoplastic pancreas $(\mathrm{n}=3)$ & 0 \\
\hline
\end{tabular}

Values in parentheses are percentages. $\mathrm{p}<0.001$.

its staining expression displaying a progressive increase in the sequence of disease progression, i.e. normal, pancreatitis and PDAC, suggesting that Galectin-3 is intimately involved in the pathogenesis of PDAC (fig. 3a-d).

In sharp contrast to the significantly elevated Galectin-3 levels in PDAC, no Galectin-3 positivity was detected in the 5 PNEN CB (fig. 4a), and 13 GIST CB (fig. 4b). Hence this demonstrated $100 \%$ negativity for Galectin-3 staining in PNEN and GIST FNA CB, which establishes that Galectin-3 is undetectable in PNEN and GIST tumors. Scattered single cells representing macrophages and lymphocytes, however, did show focal Galectin-3 staining in these cases.

PTEN expression in tumor TMAs of PDAC was totally lost in 26 of 33 (78.79\%) cases, while adjacent benign tissue and non-neoplastic cases all retained PTEN expression (fig. 5; sensitivity $78.79 \%$; specificity $100 \%$; p $<0.01$ ).

\section{Discussion}

Diagnoses of pancreatic FNA cytology specimens can often be difficult and challenging, being limited frequently by the amount of diagnostic material and contamination of blood, mucin, small intestine or gastric epithelium, as well as degenerative necrotic debris. Furthermore, differentiating PDAC from other pancreatic neoplasms can pose an even bigger challenge on paucicellular samples. Thus, a sensitive and specific tumor marker will greatly facilitate the cytologic work up of pancreatic FNAs, especially when evaluating small amounts of diagnostic material [21].

In this study, we detected that the expression of Galectin-3 is elevated in PDAC tumor tissues in FNA CB, but not in PNEN and GIST. It is also absent in the non-neoplastic pancreas and tumor-adjacent tissues. These findings were confirmed in PDAC TMA of resection specimens. Our findings also demonstrate that tumor suppressor PTEN is lost in the majority of PDAC. This is in 
Fig. 4. Immunohistochemical study of Galectin-3 levels in FNA specimens of PNEN (a, b) and GIST (c, d). HE (a, c); negative stain of Galectin-3 was observed in all cases (b, d).

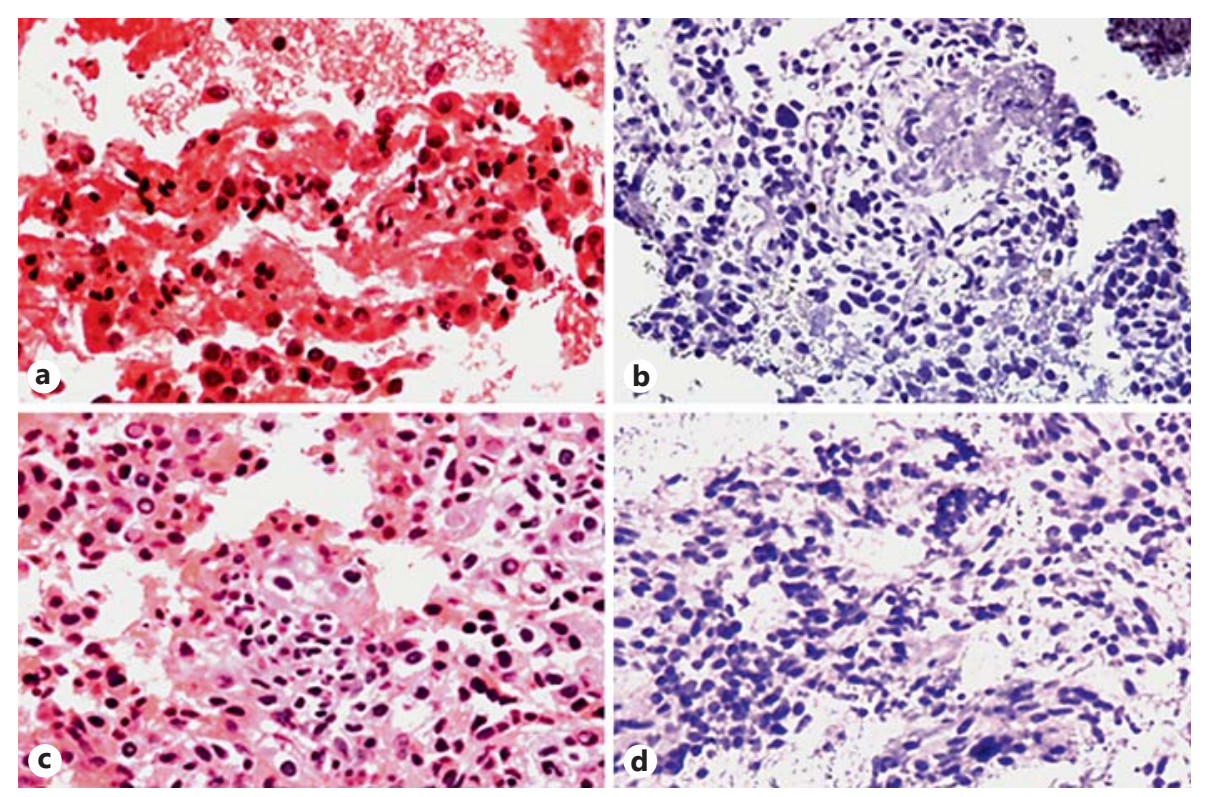

agreement with previous findings that PTEN expression is decreased in human PDAC $[22,23]$. It also suggests that elevated Galectin-3 in PDAC, but not PNEN and GIST, could be utilized in differentiating PDAC from the latter two, especially in challenging FNA cases when only limited material is available for special studies. Furthermore, PTEN could also be used to differentiate benign and reactive pancreatic tissue from PDAC.

Galectin-3 has been reported to be upregulated in a variety of human malignancies, such as gastric, colonic and thyroid carcinomas $[6-8,11]$. Although the levels of Galectin-3 have been previously reported to be elevated in PDAC compared with benign pancreatic parenchyma, the clinical significance of this finding remains undetermined [13-15]. In a recent study, Galectin-3 was demonstrated to be significantly elevated in human and mouse PDAC cells, and that it associates with oncogenic K-Ras directly and contributes to K-Ras activation in pancreatic cancer cells [4]. Elevated Galectin-3 further increases mutant K-Ras activity by recruiting K-Ras to the plasma membrane, leading to deregulated activation of downstream effectors such as Raf/MEK/ERK and PI3K/AKT molecules [4]. These findings suggest a potent role for Galectin-3 in the initiation and possibly the maintenance of malignant behavior in PDAC. Furthermore, a recent report also showed that transient silencing of Galectin-3 suppresses PDAC malignant behavior [24]. This information further indicates a functional role for Galectin-3 in PDAC tumor progression and invasion.

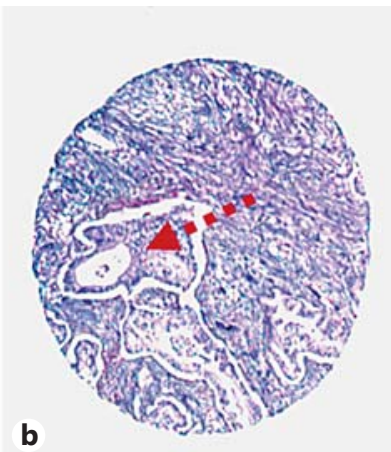

a
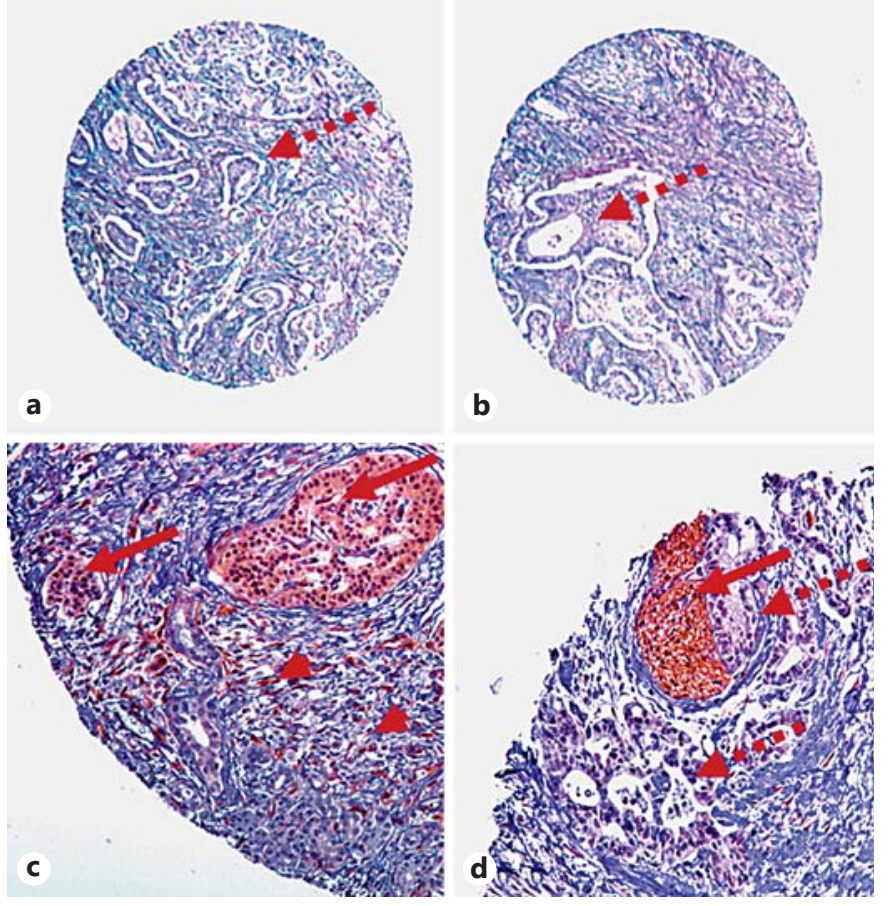

Fig. 5. Immunohistochemical study of PTEN levels in PDAC TMA. Negative PTEN stain was observed in virtually all cancerous tissue (a-d, broken arrows). c, d Focal positive stain of PTEN in islets of Langerhans (c, solid arrow), nerve (d, solid arrow) and scattered inflammatory cells (c, arrow head). Note the negative staining of tumor cells invading into and adjacent to nerves (d, broken arrows). 
The molecular mechanisms by which Galectin-3 is upregulated in PDAC are unclear. Remarkably, both mutations in K-Ras and high levels of Galectin-3 are near universal in PDAC. Galectin-3 binds oncogenic K-Ras in PDAC and induces its full activation [4]. Similarly, Galectin-3 contributes to the development of carcinoma by associating with and inducing the activation of K-Ras in thyroid gland and breast ductal epithelium $[4,25,26]$. It is well known that K-Ras mutations are early and prevalent genetic events in stimulating PDAC development. However, low levels of mutant K-Ras activity may not be sufficient to initiate PDAC tumorigenesis, which can also be observed in chronic pancreatitis and some normal pancreases [20].

Our study provides clear evidence for abnormally elevated Galectin-3 levels both in CB and TMA of PDAC samples, supporting the concept that it is involved in tumor initiation and progression, and further suggesting its potential applicability to cytopathologist diagnoses of challenging FNA cases. Our results indicate that in contrast to the aberrantly elevated Galectin-3 levels, loss of PTEN expression is an event frequently occurring in PDAC, implicating deregulated PTEN function and sig- naling in the pathogenesis and probably also the progression of PDAC. The potential additional role these markers may have in FNA samples is by their utilization for determining therapeutic options. Using TMAs prepared from resection specimens of PDAC, we have confirmed this aberrant upregulated Galectin-3 in PDAC; furthermore, we have identified that the majority of PDAC cases do not display detectable levels of PTEN. Our future work will clarify whether there is a reversed correlation between the levels of Galectin-3 and PTEN, and whether elevated Galectin-3 is involved in the downregulation of PTEN.

In conclusion, our study indicates that Galectin-3 is uniquely and highly expressed in FNA and surgical resection specimens of PDAC, but not PNEN and GIST, implicating a pivotal role played by Galectin-3 in the pathogenesis of pancreatic cancer. Our data also demonstrate that loss of PTEN is a frequent event in PDAC. Therefore, both Galectin-3 and PTEN may be used in challenging cytologic and biopsy pancreas specimens, especially when the amount of diagnostic material becomes an issue, and differentiation of PDAC from other tumors such as PNEN and GIST becomes imperative.

\section{References}

1 Liu FT, Rabinovich GA: Galectins as modulators of tumour progression. Nat Rev Cancer 2005;5:29-41.

$>2$ Dumic J, Dabelic S, Flogel M: Galectin-3: an open-ended story. Biochim Biophys Acta 2006;1760:616-635.

-3 Levy R, Biran A, Poirier F, Raz A, Kloog Y: Galectin-3 mediates cross-talk between $\mathrm{K}$ Ras and Let-7c tumor suppressor microRNA. PLoS One 2011;6:e27490.

4 Song S, Ji B, Ramachandran V, Wang H, Hafley $\mathrm{M}$, Logsdon C, Bresalier RS: Overexpressed galectin-3 in pancreatic cancer induces cell proliferation and invasion by binding Ras and activating Ras signaling. PLoS One 2012;7:e42699.

-5 Tong Z, Kunnumakkara AB, Wang H, Matsuo Y, Diagaradjane P, Harikumar KB, Ramachandran V, Sung B, Chakraborty A, Bresalier RS, Logsdon C, Aggarwal BB, Krishnan S, Guha S: Neutrophil gelatinase-associated lipocalin: a novel suppressor of invasion and angiogenesis in pancreatic cancer. Cancer Res 2008;68:6100-6108.

6 Kim SJ, Choi IJ, Cheong TC, Lee SJ, Lotan R, Park SH, Chun KH: Galectin-3 increases gastric cancer cell motility by up-regulating fascin-1 expression. Gastroenterology 2010;138: 1035-1045.

7 Shimamura T, Sakamoto M, Ino Y, Shimada K, Kosuge T, Sato Y, Tanaka K, Sekihara H, Hirohashi S: Clinicopathological significance of galectin-3 expression in ductal adenocarcinoma of the pancreas. Clin Cancer Res 2002; $8: 2570-2575$

$>8$ van den Brule F, Califice S, Castronovo V: Expression of galectins in cancer: a critical review. Glycoconj J 2004;19:537-542.

$\checkmark 9$ Song S, Mazurek N, Liu C, Sun Y, Ding QQ, Liu K, Hung MC, Bresalier RS: Galectin-3 mediates nuclear $\beta$-catenin accumulation and Wnt signaling in human colon cancer cells by regulation of glycogen synthase kinase-3 $\beta$ activity. Cancer Res 2009;69:13431349.

10 Yang RY, Rabinovich GA, Liu FT: Galectins: structure, function and therapeutic potential. Expert Rev Mol Med 2008;10:e17.

11 Berberat PO, Friess H, Wang L, Zhu Z, Bley T, Frigeri L, Zimmerman A, Buchler MW: Comparative analysis of galectins in primary tumors and tumor metastasis in human pancreatic cancer. J Histochem Cytochem 2001; 49:539-549.

12 Piccolo E, Tinari N, Sameraro D, Traini S, Fichera J, Cumashi A, La Sorda R, Spinella F, Bagnato A, Lattanzio R, D’Egidio M, Di Risio A, Stampolidis P, Piantelli M, Natoli C, Ulrich A, Iacobelli S: LGALS3BP, lectin galactoside-binding soluble 3 binding protein, induces vascular endothelial growth factor in human breast cancer cells and promotes angiogenesis. J Mol Med (Berl) 2013;91:8394.
13 Grutzmann R, Pilarsky C, Ammerpohl O, Luttges J, Bohme A, Sipos B, Foerder M, Alldinger I, Jahnke B, Schackert HK, Kalthoff H, Kremer B, Klopel G, Saeger HD: Gene expression profiling of microdissected pancreatic ductal carcinomas using high-density DNA microarrays. Neoplasia 2004;6:611-622.

14 Khayyata S, Basturk O, Adsay NV: Invasive micropapillary carcinomas of the ampullopancreatobiliary region and their association with tumor-infiltrating neutrophils. Mod Pathol 2005;18:1504-1511.

-15 Terris B, Blaveri E, Crnogorac-Jurcevic T, Jones M, Missiaglia E, Ruszniewski P, Sauvanet A, Lemoine NR: Characterization of gene expression profiles in intraductal papillary-mucinous tumors of the pancreas. Am J Pathol 2002;160:1745-1754.

16 Wang L, Friess H, Zhu Z, Frigeri L, Zimmermann A, Korc M, Berberat PO, Buchler MW: Galectin-1 and galectin-3 in chronic pancreatitis. Lab Invest 2000;80:1233-1241.

17 Jemal A, Siegel R, Xu J, Ward E: Cancer statistics 2010. CA Cancer J Clin 2010;60:277300.

18 Logsdon CD, Ji B: Ras activity in acinar cells links chronic pancreatitis and pancreatic cancer. Clin Gastroenterol Hepatol 2009; 7(suppl 11):S40-S43.

19 Takaori K, Hruban RH, Maitra A, Tanigawa $\mathrm{N}$ : Pancreatic intraepithelial neoplasia. Pancreas 2004;28:257-262. 
20 Andea A, Sarkar F, Adsay NV: Clinicopathological correlates of pancreatic intraepithelial neoplasia: a comparative analysis of 82 cases with and 152 caseswithoutpancreaticductaladenocarcinoma. Mod Pathol 2003;16:996-1006.

21 Carrara S, Cangi MG, Arcidiacono PG, Perri F, Petrone MC, Mezzi G, Beomo C, Talarico A, Cin ED, Grassini G, Doglioni C, Testoni PA: Mucin expression pattern in pancreatic diseases: findings from EUS-guided fine-needle aspiration biopsies. Am J Gastroenterol 2011;106:1359-1363.
22 Foo WC, Rashid A, Wang H, Katz MH, Lee JE, Pisters PW, Wolff RA, Abbruzzese JL, Fleming JB, Wang H: Loss of phosphatase and tensin homolog expression is associated with recurrence and poor prognosis in patients with pancreatic ductal adenocarcinoma. Hum Pathol 2013;44:1024-1030.

23 Verma A, Guha S, Wang H, Fok JY, Koul D, Abbruzzese J, Mehta K: Tissue transglutaminase regulates focal adhesion kinase/AKT activation by modulating PTEN expression in pancreatic cancer cells. Clin Cancer Res 2008; 14:1997-2005.

24 Kobayashi T, Shimuna T, Yajima T, Kubo N, Araki K, Tsutsumi S, Suzuki H, Kuwano H, Raz A: Transient gene silencing of galectin-3 suppresses pancreatic cancer cell migration and invasion through degradation of $\beta$-catenin. Int J Cancer 2011;129:2775-2786.
5 Levy R, Grafi-Cohen M, Kraiem Z, Kloog Y: Galectin-3 promotes chronic activation of $\mathrm{K}$ Ras and differentiation block in malignant thyroid carcinomas. Mol Cancer Ther 2010;9: 2208-2219.

26 Ashery U, Yizhar O, Rotblat B, Elad-Sfadia G, Barkan B, Haklai R, Kloog Y: Spatiotemporal organization of Ras signaling: rasosomes and the galectin switch. Cell Mol Neurobiol 2006; 26:471-495. 